\title{
Qualitative analysis of the health system effects of a community-based malaria elimination program in Rwanda
}

This article was published in the following Dove Press journal: Research and Reports in Tropical Medicine

\author{
Ibukun-Oluwa Omolade \\ Abejirinde' \\ Chantal Marie Ingabire ${ }^{2}$ \\ Michele van Vugt ${ }^{3}$ \\ Leon Mutesa ${ }^{4}$ \\ Bart van den Borne ${ }^{2}$ \\ Jamiu O Busari ${ }^{5,6}$
}

'Athena Institute for Research on Innovation and Communication in Health and Life Sciences, Vrije Universiteit Amsterdam, Amsterdam, the Netherlands; ${ }^{2}$ Department of Health Promotion, Maastricht University, Maastricht, the Netherlands; ${ }^{3}$ Academic Medical Center, University of Amsterdam, Amsterdam, the Netherlands; ${ }^{4}$ College of Medicine and Health Sciences, University of Rwanda, Kigali, Rwanda; ${ }^{5}$ Faculty of Health Medicine and Life Sciences, Maastricht University, the Netherlands; ${ }^{6}$ Zuyderland Medical Centre, Heerlen, the Netherlands

Correspondence: Ibukun-Oluwa Omolade Abejirinde Athena Institute for Research on Innovation and Communication in Health and Life Sciences, Vrije Universiteit Amsterdam, De Boelelaan I 105, WN Building, Room S-544, Amsterdam, I08IHV, the Netherlands

$\mathrm{Tel}+3$ I 205983 I 43

Fax +3 | 20598703 I

Email i.o.abejirinde@vu.nl
Purpose: To identify the health system-strengthening role of a community-based malaria elimination program in Ruhuha, Rwanda, and the ways by which health system effects may have been achieved.

Materials and methods: Qualitative data were collected through 14 semi-structured in-depth interviews and five focus group discussions with various stakeholders. These data were supported by analysis of project documents.

Results: Use of a transdisciplinary approach allowed the program to influence several crosscutting issues spanning four broad areas - social collaboration; capacity building; structural alignment; and knowledge translation. Health system effects were identified mostly at the micro (i.e., district) level, with limited impact on strengthening national and subnational policies. Although systems thinking was not explicitly applied, the project had positive spillover effects on the health system. These include expanding the informal health workforce and introducing innovative approaches aligned to the national malaria strategy for vector control. Findings also show that the elimination program contributed to an increased understanding of the transmission dynamics of malaria in Ruhuha.

Conclusion: The community-based malaria elimination program in Ruhuha successfully created a stable foundation for community mobilization toward malaria control, and explored innovative ways for long-term financing for malaria elimination. The transdisciplinary nature of the project, use of horizontal facilitation techniques for community engagement, and the sociocultural context in which the program was implemented are possible mechanisms by which systems strengthening was achieved. The knowledge gained from this assessment can be used to improve future community-focused interventions for malaria control, and develop a sustainable strategy for community engagement in health care.

Keywords: community-based participatory research, vertical programs, transdisciplinary research, LMIC

\section{Introduction}

Malaria is an infectious disease with high morbidity and mortality rates, caused by a mosquito-borne parasitic protozoan - Plasmodium falciparum. Although malaria is preventable and curable, it remains a significant challenge to public health and socio-economic development, especially in developing countries. Out of over 3 billion people at risk globally, the World Health Organization (WHO) reported 212 million new cases of malaria and 429,000 malaria-related deaths in $2015 .{ }^{1}$ Of these numbers, Africa had the highest burden, accounting for $92 \%$ of global malaria 
mortality, with children under 5 years representing about $70 \%$ of global malaria mortality.

To date, only a few low- and middle-income countries (LMIC) are on track toward malaria elimination. Mauritius, for example, is the only country in Sub-Saharan Africa that has successfully eliminated malaria. ${ }^{2}$ This lag in progress toward malaria elimination is partly explained by fragile national health systems and the limited capacity of countries to adequately meet the health care needs of their population..$^{3-5}$ In addition to the inability of national malaria programs to scale up and maintain progress achieved due to contextual, financial, and institutional limitations, the rising resistance to drugs and insecticides poses further challenges. ${ }^{6,7}$

Up till now, to address issues like malaria, health strategists have tended to employ disease-specific programs that are commonly described as "vertical" because they are often isolated from other public health initiatives. Conversely, "horizontal" interventions use a systemic, integrated approach and are based on Alma Ata principles. ${ }^{8}$ While the global eradication of small pox is a success story for proponents of vertical programs, there are documented concerns about their limited ability to strengthen health systems. ${ }^{9}$ System fragmentation, the introduction of parallel systems for funding, service delivery, accountability, and resource shifting, have all been described as unwanted consequences of vertical programs. ${ }^{4}$ Because weak health systems are a barrier to the successful integration of interventions into mainstream health structures, the system-wide effects of vertical interventions renew the need for making health systems strengthening a priority. ${ }^{10,11}$ The goal of health system strengthening (HSS) is to

improve the six health system building blocks [i.e., service delivery; health workforce; health information; medical technologies; health financing; leadership and governance] and manage their interactions in ways that achieve more equitable and sustained improvements across health services and health outcomes. ${ }^{5}$

\section{Malaria elimination in Rwanda}

From 2006 to 2011, the eastern African country of Rwanda recorded an $86 \%$ reduction in malaria incidence, a $74 \%$ reduction in malaria mortality, and a $71 \%$ reduction in malaria test positivity rate. ${ }^{12}$ In 2013, almost 1 million cases of malaria were reported, with higher prevalence in rural areas, and an unexplained surge of cases since 2011. ${ }^{12,13}$ As a result, the Rwandan government embarked on a revision of its malaria control strategy, in response to the changing epidemiologic landscape of malaria, and aimed to achieve pre-elimination by $2017 .^{14}$
The Ruhuha sector in Rwanda is one area burdened by malaria prevalence, with an estimated slide positivity rate of $5 \% .{ }^{15}$ Located in Bugesera district of the Eastern province, household survey results conducted in Ruhuha classify it as hypoendemic for malaria, with cases clustered around marshlands. ${ }^{15}$ Individuals from households with high socioeconomic status have a lower risk of contracting malaria. As mandated by the National Malaria Control Program (NMCP), indoor residual spraying is carried out biannually, but Ruhuha is currently not under active malaria surveillance.

\section{The Malaria Elimination Program for Ruhuha (MEPR)}

In 2012, a 4-year community-based project tagged "Malaria Elimination Program for Ruhuha (MEPR)" was launched in Ruhuha. The project team comprised of two change management specialists, four doctoral students with two supervisors each, and a project manager. A tree diagram showing the scope of project collaboration, and coverage is presented in Figure S1.

The multi-disciplinary intervention comprised of four pillars, namely:

i) a behavioral science - for social mobilization toward malaria elimination;

ii) biomedical science - for epidemiological studies and the generation of malariometric data;

iii) medical entomology - focusing on integrated vector management;

iv) health financing - which involved an assessment of community attitudes to investing in malaria elimination.

The MEPR project activities also included the renovation of some buildings and installation of equipment to support the laboratory and administrative units. This was done to support health service delivery at the Ruhuha health center. Other details about the project and findings from project pillars have been published elsewhere. ${ }^{15-19}$

The 4-year intervention was implemented following the hypothesis that integrated participation and investment of the population is crucial for achieving malaria elimination and strengthening the health system. Horizontal participatory methods using facilitation techniques and organizational change management approaches were, therefore, employed for community mobilization. ${ }^{18}$ By actively stimulating the participation and investment of the community, the MEPR sought to complement existing social and institutional structures and enhance uptake of the services already provided by the centrally organized, top-down National Malaria Control 
Program (NMCP). The anticipated overall impact of this intervention was to influence an adaptation of national and subnational health systems and policies.

The objective of this study was to identify how the MEPR project may have contributed to health system strengthening and investigate how to adapt vertical programs for system-wide effects. Specifically, it investigated the influence of the four MEPR project pillars on corresponding building blocks of the Rwandan health care system and how the intervention integrated into the existing policy and socio-environmental structures of Rwanda for malaria elimination. The central research question was "How did the community-based malaria elimination program strengthen the health system of Rwanda for malaria elimination?"

\section{Materials and methods Study site}

Ruhuha is divided into five administrative cells and 35 villages, and covers an area of $54 \mathrm{~km}^{2}$ with a population of 19,606 and 4,279 households. Rice farming is the primary agricultural practice; $77 \%$ of heads of households are reported to be rice farmers, and the majority (79\%) of houses are made of earth, clay, or dung, reflecting low socioeconomic status. ${ }^{15}$ The sector has one health center staffed by 33 personnel, including 14 nurses and three laboratory technicians.

\section{Study design and research framework}

A widely used strategy for conceptualizing health systems strengthening in different national contexts is the World Health Organization (WHO) building blocks framework. It focuses on the six building blocks of health systems and examines them against the expected goals of improved level and equity of health, responsiveness, efficiency, and financial risk protection. ${ }^{5}$ See Figure S2 for an outline of the framework.

The framework has been lauded for its functionality in affording a universal approach to describe and analyze health systems, but has been equally criticized as being too simplistic for conducting impact assessments of health systems. ${ }^{20}$ Through its four project pillars, the MEPR directly carries out actions related to four of the six WHO building blocks (i.e., health workforce, health financing, medicines and technology, and health information). Because of its proven strengths and close alignment to the MEPR project pillars, the WHO conceptual framework was applied in this qualitative study.

\section{Data collection}

Primary data were collected through 14 semi-structured indepth interviews with key informants at the project, national, district, and community levels over a 5-week period (June 1-July 6, 2015). Informants were identified purposively in consultation with the MEPR project team. The goal was to ensure representativeness such that two to five key informants with knowledge of each component of the six health systems building blocks and involvement in the MEPR were interviewed. Five focus group discussions (FGDs) with specific stakeholder groups (project supervisors, lay community, local authority, members of the community malaria action teams [CMATs], and health professionals) were also conducted (Table 1).

Interviews aided an understanding of the processes negotiated for program action, explored the perceptions of respondents on the effects of the intervention on the health system, challenges (social, institutional, infrastructural) encountered during project activities, as well as opportunities for policy uptake. FGDs used a semi-structured question guide and involved 8-12 people per session, lasting 90-120 min. All FGDs, except that with the project team, were conducted in the Kinyarwanda language by an expert facilitator. Individual interviews lasted between $30 \mathrm{~min}$ and $2 \mathrm{~h}$, and were conducted in the English language, except where respondents preferred communicating in Kinyarwanda or French, in which case the second author (CMI), a doctoral researcher, assisted as a translator. Informed consent was received (verbal or in written, as approved by the Rwanda National Ethics Committee) and documented, after explaining the purpose of the research, confidentiality, and non-attribution. All interviews except one were audio-recorded and later transcribed in the English language. Handwritten notes were taken for the interview that was not audio-recorded.

To aid interpretation of findings and provide relevant information on intervention context and on system-wide effects of the MEPR, secondary data were collected through document analysis. Critical documents reviewed are listed in Tables S1 and S2.

Table I Summary of interviews at various health system levels

\begin{tabular}{lll}
\hline Key informants' levels & $\begin{array}{l}\text { In-depth } \\
\text { interview }\end{array}$ & $\begin{array}{l}\text { Focus group } \\
\text { discussion }\end{array}$ \\
\hline Project & 5 & 1 \\
National & 3 & 0 \\
District & 6 & 2 \\
Community & 0 & 2 \\
Total & 14 & 5 \\
\hline
\end{tabular}




\section{Data analysis}

Data from the interviews and FGDs were analyzed iteratively using the five-stage framework approach for thematic analysis, as prescribed by Pope et al, ${ }^{21}$ with the WHO building blocks framework guiding the themes. The multiplicity of information sources enabled triangulation of findings, and data were coded using a deductive approach. No qualitative data analysis software was used because the transcripts and data could be conveniently and efficiently managed and analyzed manually.

\section{Ethical considerations}

This research represented a sub-component of the MEPR and was covered by the ethical approval of the project from the Rwanda National Ethics Committee (NHRC/2012/ PROT/0015 and No. 385/RNEC/2012). Participants were taken through an informed consent process prior to interviews and discussions. FGD participants received remuneration for transportation costs.

\section{Results}

First, narrative findings of each of the four project pillars and the WHO building blocks framework are presented, followed by findings on their system-wide effects and missed opportunities for health systems strengthening.

\section{Effects of MEPR on health system building blocks}

\section{Human resources}

Ruhuha sector has a supervised network of 105 community health workers (CHWs) who are remunerated via performance-based financing ( $\mathrm{PBF}$ ), although payouts from the Ministry of Health were reported to be irregular. Unlike activities such as routine counting of bed nets in households and antenatal care services, the diagnosis and treatment of malaria are not incentivized under the PBF scheme.

As part of the project, CMATs, made up of a trio comprising one village leader, a CHW in charge of maternal and child health, and the village youth leader, were established in each of the 35 villages in Ruhuha (i.e., a total of 105 persons). This initiative was in response to collective opinion during community dialogues that malaria elimination was not the responsibility of health workers alone. The three-person composition of CMATs was described as strategic, because each CMAT member played a recognized leadership role in their respective villages, and also especially because community members tended to ascribe a lower status to CHWs who did not hold community-level leadership roles. These power dynamics were in line with entrenched sociocultural norms regarding hierarchy, which positively influenced the response of the community to the activities of CMATs such as community sensitization on the importance of malaria, promoting consistent bed net use, and early closure of doors and windows in households.

Sometimes the people do not understand them, or rather they do not respect and follow the advice the CHWs give because they see that they are just also community members like them, so there is no hierarchy. [Health worker]

Most members of CMATs are strong people who are listened to well by citizens; whenever they stand up to talk in meetings, you find most people are eager to listen to them. So whatever they say is no doubt taken seriously. [FGD with Local Authority]

All CMATs members received 2 days of training on malaria transmission, mosquito breeding habits, how to conduct a basic entomological survey, and peri-domestic vector surveillance. While most community members said they were aware that malaria is a deadly disease, they reported gaining additional knowledge from home visits by CMATs.

They taught me the benefits of closing windows and doors early in evenings and proper use of bed nets, as a way of preventing mosquitoes. Although these measures were being taught to us even before; MEPR project emphasized it, and actively supervised us to see that we implement it. [FGD with Lay Community]

In addition to 1500 Rwandan Franc (\$2) of mobile airtime monthly, the project provided CMATs with promotional materials and reimbursed transportation costs for monthly attendance. Although the project cited the balance between sustainability and ownership as the reason for minimizing financial incentives, CMATs admitted that the incentives were inadequate.

We were thinking that, for the sustainability, it was not wise to provide salaries, because this may impede the activities when the MEPR is no longer there. Moreover, we wanted to create the spirit of ownership, where the benefits are not seen as for the project alone, rather for the health interest of the community. [Interview Project Team]

CMATs self-organized per village, met monthly with the MEPR team, and submitted written reports to the health center. Although they were initially detailed and regular, reports became monotonous or absent over time. A project member highlighted this as a challenge to effective monitoring, 
which motivated a change in the CMATs reporting system from sector to health center level. A health worker expressed concerns about the slim possibility of integrating CMATs into the formal system of health workers, since CHWs were available in sufficient numbers, and further mentioned that overlapping roles between CMATs and CHWs may lead to redundancy.

\section{Health financing}

While CMATs encouraged the uptake of community-based health insurance (CBHI) via co-operatives and local economic groups, it was not clear how the project planned to influence policy and practice for improving insurance coverage. The baseline MEPR survey in 2013 recorded only $66 \%$ of households in Ruhuha with health insurance. One explanation for this is that some individuals perceived they were placed in the wrong socio-economic class. A repeat stratification exercise was, however, being conducted nationally at the time of data collection.

Some may not afford especially due to socio-economic status. People may fall under a category where they do not belong to, and that has consequences on the amount of money they need to contribute to get health insurance. This implies that the socio-economic categorization should be repeated, and that is currently being conducted. [Interview Project Team]

Partial household insurance coverage was reportedly common, and services available through the package are not always maximized.

The MEPR explored how the positive economic benefit of rice farming, which promotes vector-breeding sites, can inform innovative long-term financing of malaria control measures. The expected outcome is that a proposed financing model will offer a way for all actors (from national to household level) to be engaged via a cost-sharing mechanism. In a first step toward developing a cost-share model, it was ascertained that rice farmers were willing to contribute up to $25 \%$ of estimated costs for spraying rice fields with the bacterial pathogen Bacillus thuringiensis israelensis (Bti). Farmers were also willing to invest the extra time and labor for them to spray Bti.

In future, we are looking to design a new financing model whereby farmers could contribute as well as the government and other community members for sustainability. But progressively, the government will phase out as the contribution from rice farmers increases. If the entire community could contribute for Bti, the financial contribution will be enough to cover the full cost without government intervention. [Interview Project Team]

\section{Health information system}

The health information pillar of the project was mostly used to feed data into other project components, and in establishing the nature of malaria epidemiology in Ruhuha. MEPR contributed to a clear understanding of the transmission dynamics of malaria in Ruhuha, by generating malariometric data crucial to arresting transmission. Researchers commented that most interventions to date have targeted indoor biting, and focused on pregnant women and children under 5 years - the recognized vulnerable groups. The baseline analysis of parasite carriage, however, revealed a high risk in males, especially those between the ages of 4-15 years.

In the follow-up survey, we went to about 1400 households and found a prevalence of about $0.4 \%$ in the 250 children we screened. The demographic is changing - the age that seems to be most at risk is actually 6-15 years, not children under five. [Interview Project Team]

During the baseline MEPR survey, households were marked and mapped for active malaria surveillance using geographic information systems. The use of this information remains limited to program planning, and has not been integrated into databases at the health center, the district hospital, or the national health information system to support active malaria surveillance. The data has, however, aided an understanding of malaria clustering in Ruhuha, and its explanatory factors.

\section{Medicines and technology}

FGDs conducted earlier in the project revealed that the single most reported factor that discourages the use of long-lasting insecticide-treated nets (LLIN) is bed bugs. This was reiterated in FGDs and interviews.

My home was infested by bedbugs. They occupied all house walls, beds and mattresses. At night, they climb the bed nets and cover all holes in the net, from where they descend to bite us. For sure the bite of bedbugs in so painful that you can't withstand it. That is why we prefer to remove the bed nets. [FGD with Lay Community]

A respondent from the NMCP credits the MEPR for revealing this previously unknown determinant of bed net usage. Based on this, steps were being taken to modify the 
national strategy on malaria information, education, and communication.

When we conducted different surveys in the past, the issue of bedbugs was not reported by the communities. They used to report classic responses such as heat when they sleep under nets. But from the reports of this project, when we investigated this issue of bedbugs, we saw that it is a country-wide issue. So now the message we are giving the community for the best usage of bed nets included cleaning, sanitation and how to control bed bugs. [NMCP]

Improved coverage of indoor residual spraying (IRS) was ascribed to the MEPR intervention.

If I give an example, we have now performed up to 13 rounds of IRS, and in the last round of IRS, Ruhuha was the first place we achieved $100 \%$ coverage of IRS since 2007. [NMCP]

Environmental management - a cost-effective way for vector control - poses a unique challenge in Ruhuha, which has five marshlands. Backed by evidence of successful interventions in Kenya and Tanzania, the MEPR conducted a pilot study on the use of Bti for larval source management - a strategy which stakeholders recognize as a valuable complement to other vector control activities. Taking a participatory and inclusive approach, the project team respected the refusal of farmers to have their farms sprayed by strangers and instead trained them to spray their fields. Feedback meetings between farmers, rice-cooperative groups, and the researchers were organized every 2 weeks, enhancing the co-production of knowledge. The MEPR strategy to stimulate policy uptake for Bti application and its integration with rice-farming practices was to be developed post-implementation by drawing recommendations from lessons learned.

\section{System-wide effects of the MEPR}

The transdisciplinary focus of the intervention was seen to enhance HSS effects. All four project pillars worked interdependently; relying on each other's activities, while keeping the community central to all actions. For example, results of FGDs carried out by the behavioral/social mobilization arm of the project facilitated problem framing for the vector management pillar. In piloting the use of a bacterial pathogen to kill mosquito larvae - Bti, the project liaised with administrative structures at the sector, district, and national levels (Executive secretary and Agronomist of the sector; Ministry of Agriculture and Environment). Therefore, by taking a transdisciplinary approach and interlinking the four pillars, the MEPR influenced several crosscutting issues spanning four broad areas - social collaboration (in the form of social value generated from partnerships with health system stakeholders); capacity building; structural alignment (i.e., integrating with existing structures); and knowledge translation (Figure 1).

At the national level, the Rwandan Biomedical Centre managed the MEPR through the Single Project Implementation Unit - a governmental anticorruption unit for donor projects. Annual dissemination meetings involving all stakeholders were organized in collaboration with a research coalition called the Knowledge Translation Network Africa that

Social collaboration
- Strengthened interaction between the health center and the community
Collaboration between international and national partners
Capacity building
- Strengthened national capacity through doctoral students whose skills and expertise
- Training of CMAT members, rice farmers, and laboratory technicians
- Enhancing infrastructural and technical capacity at Ruhuha health center and at MRC
Structural alignment
- Maximizing decentralization and aligning to community norms and values
administrative overlaps
Knowledge translation
- "Expert" knowledge has been effectively communicated to the lay community (CMATs
and rice farmers)

Figure I System-wide effects of the MEPR.

Abbreviations: MEPR, Malaria Elimination Program for Ruhuha; CMAT, community malaria action team; MRC, Medical Research Centre; CHW, community health worker. 
supports the translation of evidence to policy. Project reports and informal communication revealed that collaboration challenges with national stakeholders in the first implementation year resulted in delayed project onset. Collaboration with the local authority was, however, visibly integral to the activities of all project pillars.

The MEPR was perceived by most respondents to have helped to establish closer ties between the health facilities and the local community. However, we identified missed opportunities by the MEPR for systems strengthening at the local, district, and national levels. These include: optimizing activities of the health financing pillar to promote coverage of community-based health insurance (local and district levels); integration of malariometric data gathered in the biomedical science pillar with the national health information system (national level); institutionalization of the CMATs into the existing formal health workforce (district level); and sustained collaboration with the national level to strengthen evidence uptake (national level).

\section{Discussion}

The extent to which a program can strengthen the health system can provide clues to its ability to integrate with and complement other public health interventions. The MEPR aimed to contribute to malaria pre-elimination in Rwanda by connecting community knowledge and resources to the national malaria control program through social mobilization, while strengthening the health system for malaria control. Although the body of knowledge on how a health system strengthening agenda can be successfully integrated and implemented into vertical programs is still growing, ${ }^{4,22}$ this interim analysis of the MEPR has provided some insight on how and to what extent HSS worked in practice (Figure 2), including missed opportunities to be considered by the project when revising the implementation strategy.

The project strengthened workforce capacity for malaria action through increased coverage of information, education, and communication activities via CMATs who focused on malaria prevention and control. This did not seem to overlap with the activities of CHWs who concentrated on malaria diagnosis and treatment, therefore minimizing potential conflict. Although the CMATs network was not considered disruptive to the existing $\mathrm{CHW}$ system and it ensured increased community participation in the project, a lack of institutionalization and formalization of CMATs roles reflected poor integration into the mainstream community health system of Ruhuha. Since CMATs already represent establish district administrative structures (i.e., the village leader, youth leader, etc.), their integration into the formal health workforce would have allowed the skills acquired under the MEPR to be employed in other diseases prevalent in Ruhuha such as malnutrition. This was the case in a study that promoted integrated service delivery for childhood diseases through volunteer health workers, therefore stimulating workforce

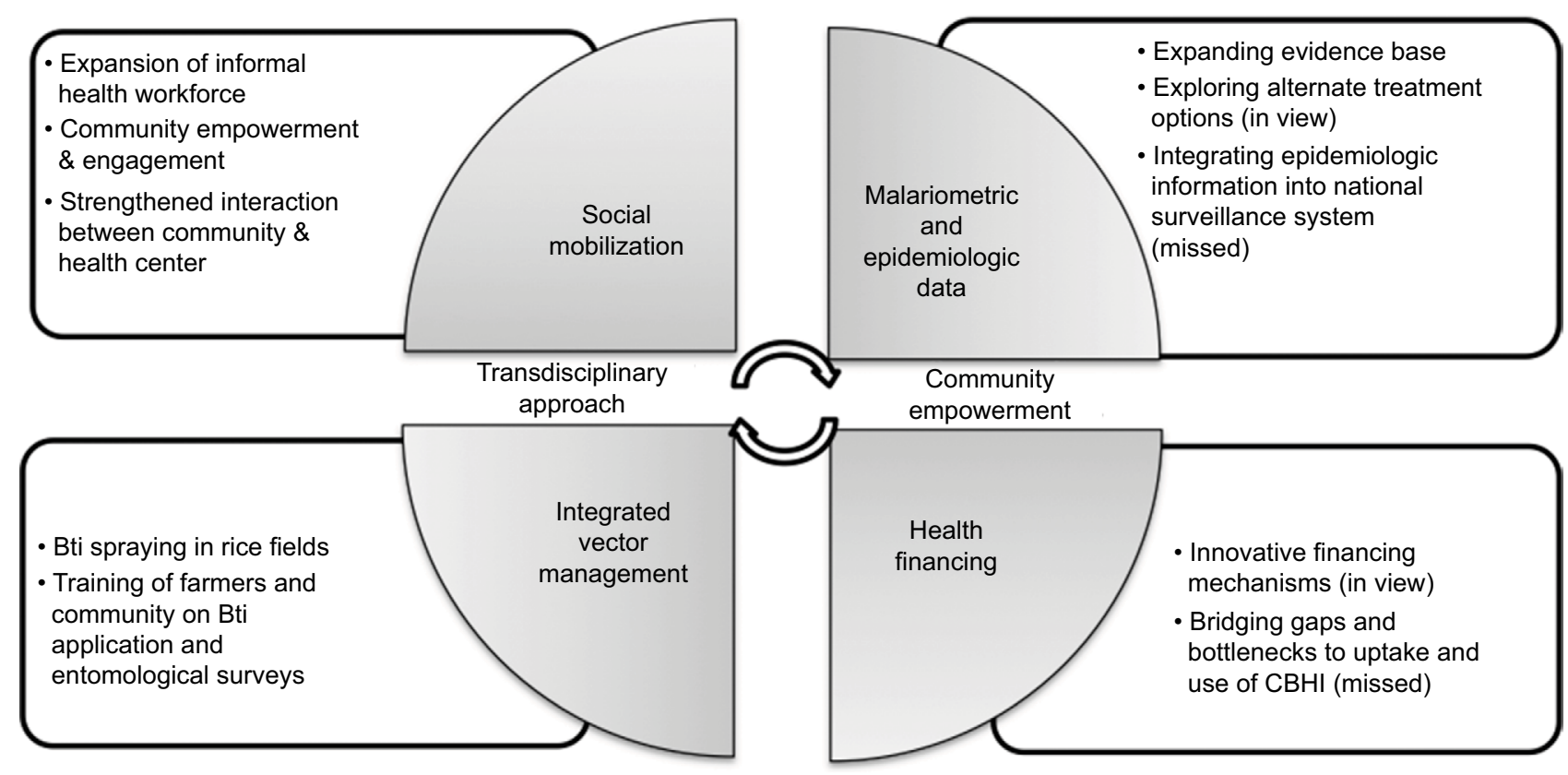

Figure 2 Health systems strengthening effects of the different MEPR pillars (including missed opportunities).

Abbreviations: MEPR, Malaria Elimination Program for Ruhuha; Bti, Bacillus thuringiensis israelensis; $\mathrm{CBHI}$, community-based health insurance. 
expansion. ${ }^{23}$ How to efficiently ensure motivation and create incentives for these workers remains open to investigation, but lessons can be learned from similar efforts. ${ }^{24,25}$ Interestingly, a multi-country study on community-led interventions reported non-financial incentives as being more effective in motivating community volunteers. ${ }^{26}$ By establishing processes that are not heavily dependent on direct financial motivation, there is also a higher chance of sustainability.

Community engagement, including the formation of village committees, has been similarly identified as a crucial strategy for effective community-based primary care programs. ${ }^{27}$ Such participatory approaches strengthen commitment and program sustainability. ${ }^{26}$ Concerns have, however, been raised about the quality of training these cadres of workers receive, and its implications for quality of health services, if not embedded in robust health systems and supervisory structures. ${ }^{28,29}$

Activities of the integrated vector management (IVM) pillar were one of the most reliable HSS components of the MEPR and were aligned to the national strategy for malaria pre-elimination. The pillar adopted a number of best-practices (capacity building, adaptation to local vector ecology and resources, multisectoral collaboration) within a health systems lens, as defined by the global strategic framework for IVM..$^{30,31}$ However, interlinkages with the national level were mostly procedural - vis-à-vis signing a memorandum and submitting annual reports - and to a lesser extent collaborative and integrated. This could have been due to initial challenges encountered during the first phase of implementation, and the projects' focus on community engagement, which did not initially allow for the continued involvement of policy level stakeholders. Lessons can be learned from the vector management strategy in Zambia, which had elements of strong community engagement and national consolidation. ${ }^{32}$ The importance of early and continued engagement with policy level stakeholders cannot be underemphasized in any program geared to effectively strengthen the health system. ${ }^{33} \mathrm{~A}$ similar analysis of the effect of malaria programs in two Asian countries identified gaps between the programs and the health systems, despite good collaboration at the national level ${ }^{34}$ Given the long-term goal to institutionalize the participatory approach, despite the fact that the MEPR focused more on the district and sector levels, the top-down hierarchical structure of the national system in which it operated should not be ignored. ${ }^{35}$ This is because weak or unsustained linkages between the project and national stakeholders could delay eventual uptake of evidence.
By harnessing resources available at the health facility and in the community, the MEPR partially integrated with institutional structures at the primary health level. Health service delivery to the community also experienced capacity building through technical and infrastructural support. Leadership and governance structures in the health system were influenced mainly at the level of the community, which played a central role in MEPR activities, therefore allowing for bottom-up strengthening. ${ }^{36}$ Because it leverages existing resources and capabilities while empowering actors, the strengthening of community systems via community participation in health is recognized as indispensable to the primary health care model. ${ }^{24}$ Formative assessment of the project revealed other health system bottlenecks such as insufficient insurance coverage, low level of trust for services offered at the health center, and factors limiting the use of LLIN such as reports of bed-bug infestations linked to net use. ${ }^{19}$ Such resource and logistical challenges that limit the impact of malaria control programs are not unique to our study. ${ }^{34,35}$ Given limitations of finances and time, the intended effects of the MEPR on the six health system building blocks were not as substantial as initially envisioned.

Although health systems are popularly viewed through conceptual frameworks such as the building blocks framework, this analysis supports the view that it is people (in this case the Ruhuha community) at the core of the building blocks, who give meaning and drive to the health system. ${ }^{9,37}$ The use of facilitation techniques based on the principle of holocracy (i.e., redistributive authority and joint learning in self-organized teams) against the background of the viable institutional and socio-cultural context in Rwanda, could explain the unintended mechanisms by which identified health systems strengthening effects were achieved by the MEPR. While this report does not elaborate on this process, the initial steps have been published as part of the project output. ${ }^{18}$

According to the WHO, health systems challenges for a malaria pre-elimination program include engaging the private sector, dealing with over-the-counter sale of medicines, and ensuring adequate human resources. ${ }^{38}$ Although researchers in the MEPR project seemed to have a systemic approach in mind, there was no clear strategy on how pillar activities would strengthen the health system. Project impact could, therefore, have been enhanced if planning had included an initial rapid systemic analysis, applying systems thinking prospectively not retroactively. There are, however, potential practical difficulties to consider when using systems thinking. These include agreement on what systems thinking is, and 
how it should be applied in strengthening the health system and managing the divergent views and expectations of all stakeholders. ${ }^{33}$

Despite the limitations, a core component of the MEPR was its innovative approach in facilitating community empowerment for health systems strengthening. In this way, the project showed characteristics of a hybrid; a vertical intervention with a bottom-up approach anchored on community ownership. Therefore, although a systems approach was not explicitly applied during the intervention, our analysis shows that it attained some system strengthening effects, mostly revealed in the social mobilization and integrated vector management pillars.

\section{Limitations}

This paper has served to contribute to the knowledge of how health systems strengthening may be achieved in diseasespecific programs, using malaria control in the Ruhuha sector, Rwanda as a case study. However, it should not be interpreted as a comprehensive health systems analysis, because the project was restricted to the Ruhuha sector, of which this study reports an interim assessment. The observed system strengthening effects should, therefore, be interpreted within the context of the district or community health system. Additionally, the extent to which health system strengthening effects may be directly attributed to the MEPR project could not be established, due to a lack of comparable data from other malaria-endemic communities. Other factors, including limited scope and resources, did not allow us to explore the synergistic effect of this intervention alongside other programs occurring in the same context.

\section{Conclusion}

The MEPR effectively mediated community engagement in the Ruhuha health system, specifically for malaria control. It had significant effects on the health system of the Ruhuha sector by expanding the informal health workforce, and it influenced the central level of the health system by introducing innovative vector control techniques aligned to the national strategy for malaria control. However, its aim to influence the health system was limited to the micro-level, such that opportunities to stimulate and strengthen national and sub-national policies were not fully optimized. Although a holistic and fully integrated health systems strengthening project is ambitious for a 4-year timeline, the MEPR successfully scratched the surface by identifying challenges and opportunities which influence malaria control strategy, setting local precedence for community mobilization toward malaria control, and exploring innovative ways for financing malaria initiatives.

\section{Acknowledgments}

We acknowledge the MEPR implementation team and all study respondents, without whom data collection would not have been possible. We would like to thank the two anonymous reviewers for their useful suggestions and comments, which helped to improve the manuscript.

This study is a sub-component of the MEPR project funded by WOTRO Science for Global Development (Grant \# SA 358001). The project covered all data collection costs related to this paper. Funders had no role in data collection, analysis, or the decision to publish.

\section{Author contributions}

All authors contributed toward data analysis, drafting and revising the paper and agree to be accountable for all aspects of the work.

\section{Disclosure}

The authors report no conflicts of interest in this work.

\section{References}

1. World Health Organization. World Malaria Report 2015. Geneva, Switzerland: WHO; 2016. Available from: http://www.who.int/malaria/ publications/world-malaria-report-2015/WMR2015.pdf. Accessed March 9, 2017.

2. World Health Organization. World Malaria Report 2011. Geneva, Switzerland: WHO; 2012. Available from: http://www.who.int/malaria/ world_malaria_report_2011/9789241564403_eng.pdf. Accessed March 19, 2017.

3. Atun R, Lazarus JV, Van Damme W, Coker R. Interactions between critical health system functions and HIV/AIDS, tuberculosis and malaria programmes. Health Policy Plan. 2010;25:i1-i3.

4. Travis P, Bennett S, Haines A, et al. Overcoming health-systems constraints to achieve the Millennium Development Goals. Lancet. 2004;364:900-906.

5. World Health Organization. Everybody's business - strengthening health systems to improve health outcomes: WHO's framework for action. Geneva, Switzerland: WHO; 2007. Available from: http://www. who.int/healthsystems/strategy/everybodys_business.pdf. Accessed January 12, 2017.

6. World Health Organization. From Malaria Control to Malaria Elimination: A Manual for Elimination Scenario Planning. Geneva, Switzerland: WHO; 2014. Available from: http://apps.who.int/iris/bitstream/ handle/10665/112485/9789241507028_eng.pdf?sequence $=1$. Accessed January 12, 2017.

7. Mills A, Lubell Y, Hanson K. Malaria eradication: the economic, financial and institutional challenge. Malar J. 2008;7:S11.

8. Atun RA, Bennett S, Duran A. When do vertical (stand alone) programmes have a place in health systems? Copenhagen: WHO Regional Office for Europe; 2008

9. De Savigny D, Adam T. (Eds). Systems thinking for health systems strengthening. Geneva: Alliance for Health Policy and Systems Research, WHO; 2009. Available from: http://apps.who.int/iris/bitstream/handle/10665/44204/9789241563895_eng.pdf?sequence $=1$. Accessed January 12, 2017. 
10. Mills AJ, Ranson MK. The design of health systems. In: Merson MH, Black RE, Mills A, editors. International Public Health: Diseases, Programs, Systems and Policies. Boston, MA: Jones and Bartlett Publishers; 2006.

11. de Savignv D. Health systems stewards and health systems researchers: a critical partnership for malaria elimination. Malar J. 2012;11:013.

12. President's Malaria Initiative. President's Malaria Initiative. Rwanda Malaria Operational Plan FY 2014. Washington, DC: President's Malaria Initiative; 2013. Available from: https://www.pmi.gov/docs/ default-source/default-document-library/malaria-operationalplans/ fy14/rwanda_mop_fy14.pdf?sfvrsn=8. Accessed January 12, 2017.

13. World Health Organization. World Health Statistics 2015. Geneva, Switzerland: WHO; 2015. Available from: http://apps.who.int/iris/ bitstream/handle/10665/170250/9789240694439_eng.pdf?sequence=1. Accessed March 9, 2017.

14. Rwanda National Malaria Control Programme: Rwanda Malaria Control Strategic Plan July 2013-June 2018. Kigali: Ministry of Health Rwanda; 2013.

15. Kateera F, Mens PF, Hakizimana E, et al. Malaria parasite carriage and risk determinants in a rural population: a malariometric survey in Rwanda. Malar J. 2015;14:16.

16. Ingabire $\mathrm{CM}$, Hakizimana $\mathrm{E}$, Kateera $\mathrm{F}$, et al. Using an intervention mapping approach for planning, implementing and assessing a community-led project towards malaria elimination in the Eastern Province of Rwanda. Malar J. 2016;15:594.

17. Ingabire $\mathrm{CM}$, Hakizimana $\mathrm{E}$, Rulisa $\mathrm{A}$, et al. Community-based biological control of malaria mosquitoes using Bacillus thuringiensis var. israelensis (Bti) in Rwanda: community awareness, acceptance and participation. Malar J. 2017;16:399.

18. Ingabire CM, Alaii J, Hakizimana E, et al. Community mobilization for malaria elimination: application of an open space methodology in Ruhuha sector, Rwanda. Malar J. 2014;13:167.

19. Ingabire CM, Rulisa A, Van Kempen L, et al. Factors impeding the acceptability and use of malaria preventive measures: implications for malaria elimination in eastern Rwanda. Malar J. 2015;14:136.

20. Mounier-Jack S, Griffiths UK, Closser S, Burchett H, Marchal B. Measuring the health systems impact of disease control programmes: a critical reflection on the WHO building blocks framework. BMC Public Health. 2014;14:278.

21. Pope C, Ziebland S, Mays N. Qualitative research in health care: analysing qualitative data. BMJ. 2000;320:114-116.

22. World Health Organization Maximizing Positive Synergies Collaborative Group. An assessment of interactions between global health initiatives and country health systems. Lancet. 2009;373:2137-2169.

23. Brenner JL, Barigye C, Maling S, et al. Where there is no doctor: can volunteer community health workers in rural Uganda provide integrated community case management? Afr Health Sci. 2017;17:237-246.

24. Atkinson JA, Vallely A, Fitzgerald L, Whittaker M, Tanner M. The architecture and effect of participation: a systematic review of community participation for communicable disease control and elimination. Implications for malaria elimination. Malar J. 2011;10:225.
25. United States Agency for International Development. Malaria Control in Cambodia: Community Mobilization for Malaria Prevention, Diagnosis, and Treatment. Washington, DC: United States Agency for International Development; 2011. Available from: http://www.chwcentral.org/sites/ default/files/MCC_comm_mobilization_brief_final.pdf. Accessed January 12, 2017.

26. CDI Study Group. Community-directed interventions for priority health problems in Africa: results of a multicountry study. Bull World Health Organ. 2010;88:509-518.

27. Perry HB, Sacks E, Schleiff M, et al. Comprehensive review of the evidence regarding the effectiveness of community-based primary health care in improving maternal, neonatal and child health: 6 . strategies used by effective projects. $J$ Glob Health. 2017;7:010906.

28. Hermann K, Van Damme W, Pariyo GW, et al. Community health workers for ART in sub-Saharan Africa: learning from experience - capitalizing on new opportunities. Hum Resour Health. 2009;7:31.

29. Zachariah R, Ford N, Philips M, et al. Task shifting in HIV/AIDS: opportunities, challenges and proposed actions for sub-Saharan Africa. Trans R Soc Trop Med Hyg. 2009;103:549-558.

30. Beier JC, Keating J, Githure JI, Macdonald MB, Impoinvil DE, Novak RJ. Integrated vector management for malaria control. Malar J. 2008;7:S4.

31. World Health Organization. Global Strategic Framework for Integrated Vector Management. Geneva, Switzerland: WHO; 2004. Available from: http://apps.who.int/iris/bitstream/handle/10665/68624/WHO_CDS_ CPE_PVC_2004_10.pdf? sequence=1. Accessed March 9, 2017.

32. Chanda $E$, Masaninga F, Coleman M, et al. Integrated vector management: the Zambian experience. Malar J. 2008;7:164.

33. El-Jardali F, Adam T, Ataya N, Jamal D, Jaafar M. Constraints to applying systems thinking concepts in health systems: A regional perspective from surveying stakeholders in Eastern Mediterranean countries. Int $J$ Health Policy Manag. 2014;3:399-407.

34. Ohara H, Sherchan JB, Pokhrel BM, Hirayama T, Vu HN, Sherchand JB. Assessment of health systems in relation to interface between malaria control programs and health system strengthening: comparative study between Nepal and Viet Nam. $J$ Inst Med. 2015;37(1):11-20.

35. Chaki PP, Kannady K, Mtasiwa D, et al. Institutional evolution of a community-based programme for malaria control through larval source management in Dar es Salaam, United Republic of Tanzania. Malar J. 2014;13:245.

36. Taylor DC, Taylor CE. Just and Lasting Change: When Communities Own Their Futures. Baltimore: JHU Press; 2016.

37. Shakarishvili G, Atun R, Berman P, Hsiao W, Burgess C, Lansang MA. Converging health systems frameworks: towards a concepts-to-actions roadmap for health systems strengthening in low and middle income countries. Glob Heal Gov. 2010;3:1-17.

38. World Health Organization. Malaria Elimination: A Field Manual for Low and Moderate Endemic Countries. Geneva, Switzerland: WHO; 2007. Available from: https://docs.google.com/viewer? $\mathrm{a}=\mathrm{v} \&$ pid=sites\&srcid=ZGVmYXVsdGRvbWFpbnxtYXBwaW5nbWFsYXJpYXxne Do0MzFjODA2MGY0OTNhZGQ5. Accessed March 9, 2017. 


\section{Supplementary materials}

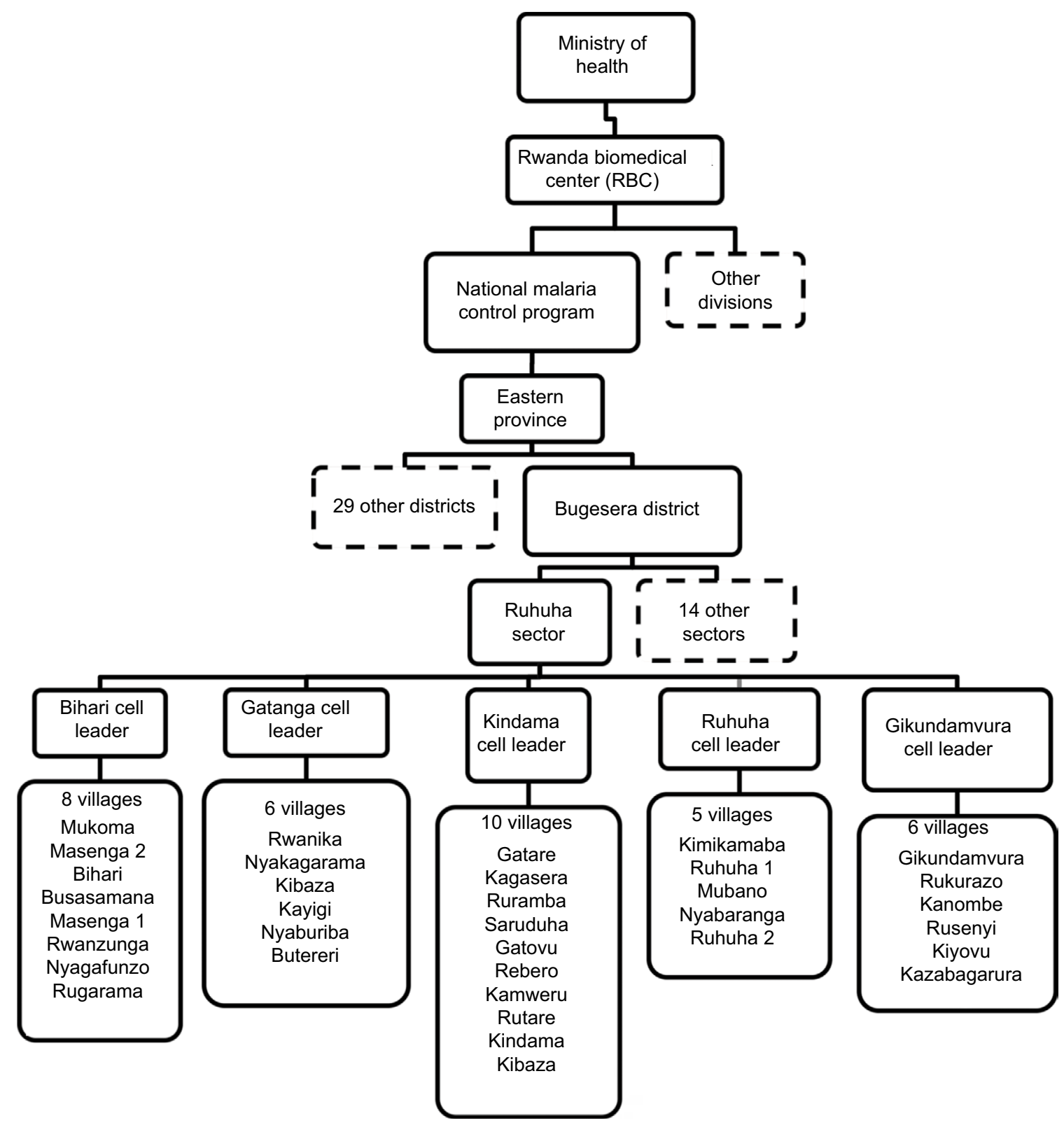

Figure SI Hierarchical scope of collaboration and coverage of the MEPR (excluding broken squares). Abbreviation: MEPR, Malaria Elimination Program for Ruhuha. 
System building blocks ${ }^{b}$

Service delivery
Health workforce
Health information
Medicinal products \& technologies
Health financing
Leadership/governance

Figure S2 The WHO systems framework. ${ }^{a}$

Notes: 'Reprinted from World Health Organization. Everybody's Business: Strengthening Health Systems to Improve Health Outcomes: WHO's Framework for Action. Geneva: World Health Organization; I-16. Available from: http://www.who.int/healthsystems/strategy/everybodys business.pdf. Copyright 2007.' bService that is provided in an effective and safe manner with adequate infrastructural support. A health workforce that is efficiently trained and is available in sufficient numbers. Health information involving the production, analysis, and dissemination of reliable information on health status, health systems performance, and health determinants. Medical products, vaccines, and technologies, which are cost-effective, accessible, safe, and are in line with norms, standards, and policies. Financing that protects health care consumers from financial impoverishment. Leadership and governance involving strategic health sector policies, harmonization, coalition building, oversight, and regulation.

Abbreviation: WHO, World Health Organization.

Table SI Other data sources for document analysis

i. Global Health Policy and Health Systems Research. Malaria Elimination Program for Ruhuha (MEPR) Full Proposal. December 2009

ii. Government of Rwanda, Ministry of Health. 3rd Health Sector Strategic Plan 20I2-20I8

iii. Malaria Elimination Program for Ruhuha (MEPR). Manual for Community Malaria Action Teams

iv. Monthly Community Malaria Action Teams Report (July 2014-February 2015)

v. National Malaria Control Program 2008

vi. Rwanda Annual Health Statistics Booklet 2013

vii. Rwanda Bti Application Protocol

viii. Rwanda Demographic and Health Survey 2005 and 2010

ix. Rwanda Malaria Control Strategic Plan (July 2013-June 2018)

x. World Bank Database

xi. WHO NHA- http://apps.who.int/nha/database

Abbreviations: Bti, Bacillus thuringiensis israelensis; NHA, National Health Accounts; WHO, World Health Organization.

Table S2 Summary of information gathered from academic output of MEPR

\begin{tabular}{|c|c|}
\hline Publication & Information \\
\hline Ingabire et $\mathrm{al}^{2}$ & $\begin{array}{l}\text { - Bedbugs and discomfort due to heat are main factors explaining gap between access and use of bed nets } \\
\text { - Lack of } \mathrm{CBHI} \text { delays health seeking behavior } \\
\text { - Malaria framed as a socio-economic problem }\end{array}$ \\
\hline Ingabire et $\mathrm{al}^{3}$ & $\begin{array}{l}\text { - Expectations and recommendations of the community for malaria control } \\
\text { - Process by which facilitatory techniques were applied and how community engagement was facilitated by the MEPR }\end{array}$ \\
\hline Kateera et $\mathrm{al}^{4}$ & $\begin{array}{l}\text { - Malaria parasitaemia found in } 5 \% \text { of individuals surveyed in Ruhuha, with } 13 \% \text { of households having at least one } \\
\text { parasitaemic member } \\
\text { - High parasite carriage risk was associated with being male and persons aged 4-I5 years } \\
\text { - Households of higher socio-economic status, with educated head of household and those constructed using cement } \\
\text { or bricks showed risk-protective effect } \\
\text { - Parasitaemia significantly clusters in marshlands }\end{array}$ \\
\hline
\end{tabular}

Abbreviations: MEPR, Malaria Elimination Program for Ruhuha; $\mathrm{CBHI}$, community-based health insurance. 


\section{References}

1. World Health Organization. Everybody's Business: Strengthening Health Systems to Improve Health Outcomes: WHO's Framework for Action. Geneva: World Health Organization; 2007. Available from: http://www. who.int/healthsystems/strategy/everybodys_business.pdf. Accessed January 12, 2017.

2. Ingabire CM, Rulisa A, Van Kempen L, et al. Factors impeding the acceptability and use of malaria preventive measures: implications for malaria elimination in eastern Rwanda. Malar J. 2015;14:136.
3. Ingabire CM, Alaii J, Hakizimana E, et al. Community mobilization for malaria elimination: application of an open space methodology in Ruhuha sector, Rwanda. Malar J. 2014;13:167.

4. Kateera F, Mens PF, Hakizimana E, et al. Malaria parasite carriage and risk determinants in a rural population: a malariometric survey in Rwanda. Malar J. 2015;14:16.

\section{Publish your work in this journal}

Research and Reports in Tropical Medicine is an international, peerreviewed, open access journal publishing original research, case reports, editorials, reviews and commentaries on all areas of tropical medicine, including: Diseases and medicine in tropical regions; Entomology; Epidemiology; Health economics issues; Infectious disease; Laboratory science and new technology in tropical medicine; Parasitology; Public health medicine/health care policy in tropical regions; and Microbiology. The manuscript management system is completely online and includes a very quick and fair peer-review system. Visit http://www.dovepress. com/testimonials.php to read real quotes from published authors.

Submit your manuscript here: https://www.dovepress.com/research-and-reports-in-tropical-medicine-journal 